\title{
Aberrant promoter hypermethylation and silencing of the critical 3p21 tumour suppressor gene, RASSF 1A, in Chinese oesophageal squamous cell carcinoma
}

\author{
MICHELLE LY WONG ${ }^{1}$, QIAN TAO ${ }^{4-6}$, LI FU ${ }^{4}$, KAI-YAU WONG ${ }^{1}$, GUO-HUA QIU ${ }^{4}$, FIAN B.F. LAW ${ }^{1}$, \\ PUI-CHI TIN ${ }^{1}$, WAI-LING CHEUNG ${ }^{1}$, PING-YIN LEE ${ }^{2}$, JOHNNY CHEUK-ON TANG ${ }^{1,7,8}$, \\ GEORGE S.W. TSAO ${ }^{3}$, KING-YIN LAM ${ }^{9}$, SIMON LAW ${ }^{2}$, JOHN WONG ${ }^{2}$ and GOPESH SRIVASTAVA ${ }^{1}$ \\ Departments of ${ }^{1}$ Pathology, ${ }^{2}$ Surgery and ${ }^{3}$ Anatomy, The University of Hong Kong, Hong Kong, P.R. China; \\ ${ }^{4}$ Johns Hopkins Singapore, Singapore; ${ }^{5}$ Sidney Kimmel Comprehensive Cancer Centre, Johns Hopkins School of Medicine, \\ Baltimore, MD, USA; ${ }^{6}$ Cancer Epigenetics Laboratory, Cancer Centre, Department of Clinical Oncology, The Chinese \\ University of Hong Kong; ${ }^{7}$ Department of Applied Biology and Chemical Technology, The Hong Kong Polytechnic \\ University; ${ }^{8}$ State Key Laboratory of Chinese Medicine and Molecular Pharmacology, Shenzhen, P.R. China; \\ ${ }^{9}$ Department of Pathology, Griffith Medical School, Griffith University, Queensland, Australia
}

Received August 11, 2005; Accepted September 23, 2005

\begin{abstract}
. 3p21 is an important locus harbouring critical tumour suppressor genes (TSG), which are implicated in the pathogenesis of multiple tumours, including oesophageal carcinoma. RASSF $1 A$ is a $3 \mathrm{p} 21.3$ candidate TSG frequently inactivated by promoter methylation in multiple tumours. We investigated RASSF1A promoter methylation and gene expression in Chinese oesophageal squamous cell carcinoma (ESCC) to compare it to data from Japanese patients. Methylation-specific PCR (MSP) showed that RASSF1A was partially methylated in 3/7 (43\%) cell lines; 22/64 (34\%) primary tumours and 3/64 (5\%) corresponding non-tumour samples; and was not methylated in 2 immortalized normal oesophageal epithelial cell lines and 6 normal oesophageal epithelium samples. Bisulfite genome sequencing confirmed the MSP results. Promoter hypermethylation correlated well with RASSF 1A mRNA down-regulation. Treatment of cell lines with 5-aza-2'-deoxycytidine activated RASSF $1 A$ mRNA expression along with promoter demethylation. RASSF $1 A$ hypermethylation in the Chinese cohort was much lower than
\end{abstract}

Correspondence to: Professor Gopesh Srivastava, Department of Pathology, The University of Hong Kong, Queen Mary Hospital, 102 Pokfulam Road, Hong Kong, P.R. China

E-mail: gopesh@pathology.hku.hk

Dr Qian Tao, Cancer Epigenetics Laboratory, Cancer Centre, Department of Clinical Oncology, The Chinese University of Hong Kong, Hong Kong, P.R. China

E-mail: qtao@clo.cuhk.edu.hk

Key words: oesophageal carcinoma, tumor suppressor gene, 3p21, RASSF1A, methylation in a published report of Japanese ESCC patients (52\%) and cell lines $(74 \%)$. Our own analysis of Japanese ESCC cell lines for direct comparison also detected a high frequency of RASSF 1A hypermethylation $(8 / 10 ; 80 \%)$ and high levels of hypermethylation at each $\mathrm{CpG}$ site. No significant association between RASSF 1A hypermethylation and histological differentiation $(\mathrm{p}=0.953)$, tumour staging $(\mathrm{p}=0.117)$, or survival $(\mathrm{p}=0.7571)$ was found in Chinese ESCC, unlike the results of Japanese patients. The incidence of oesophageal cancer shows marked variation by geographic area and ethnic group; it is almost three times higher in China than in Japan, indicating possible different pathogenetic mechanisms. Our results show that RASSF 1A hypermethylation in ESCC has epidemiological/ethnic differences, and suggest that Chinese ESCC may result from different pathogenetic mechanisms.

\section{Introduction}

Oesophageal cancer is the sixth most common cancer in males in the world. Its age-standardized incidence rate is $11.5 / 10^{5}$ males (1). It is well known for its marked variation by geographic area and ethnic group. For example, it is the fourth most common cancer in China $\left(27.4 / 10^{5}\right)$, seventh in Japan $\left(10 / 10^{5}\right)$, ninth in United Kingdom $\left(9.6 / 10^{5}\right)$ and sixteenth in United States of America $\left(5.9 / 10^{5}\right)(1)$. No molecular mechanisms have yet been demonstrated to explain these variations. There are two major histological subtypes of oesophageal cancer: oesophageal squamous cell carcinoma (ESCC), which is the predominant subtype in Orientals; and oesophageal adenocarcinoma (EADC), which is more common in Caucasians. Despite advances in multimodality therapy, ESCC remains a significant problem with very low 5-year survival rates (2).

Gene amplification and/or overexpression are frequently involved in the pathogenesis of ESCC (2). Recent application of comparative DNA fingerprinting performed by our group 
revealed that amplifications or deletions of specific chromosomal regions are common in both ESCC preneoplastic lesions and carcinomas (3). By cDNA arrays, our group and others have demonstrated that multiple cancer-related genes are differentially expressed in ESCC with several downregulated tumour suppressor genes (TSGs) (4-8).

It is well established that epigenetic and genetic alterations are central to the pathogenesis of human cancers (9). Aberrant hypermethylation of $\mathrm{CpG}$ islands in TSG promoters has been found in a variety of cancers; with some promoters hypermethylated in most tumours and others being more cancertype specific. In ESCC, frequent epigenetic inactivation by promoter hypermethylation has been documented in CDKN2 (10); FHIT (11); pI4ARF, p16INK4a and pl5INK4b (12); HLA class I (13); E-cadherin (14); O-6-methylguanine-DNA methyltransferase (15); VHL (16); RAR- $\beta(16,17) ; E C R G 4$ (18) and TSLC1 (19).

Allelic loss of 3p21 is frequent in many cancers (20), suggesting the presence of critical TSGs in this locus. RASSF 1, a candidate TSG, is located within a critical $120-\mathrm{kb}$ region at 3 p21.3 that shows frequent homozygous deletions in lung tumour cell lines $(21,22)$. RASSF1 has a predicted Ras-association domain and homology to Ras-effector NORE1A (23). RASSF1 encodes two major isoform transcripts, RASSF $1 A$ and RASSF $1 C$, derived from alternative splicing and different promoter usage. The loss of RASSF1A has been suggested to play a key role in tumourigenesis. RASSF $1 A$ is frequently inactivated by promoter hypermethylation or loss of heterozygosity ( $\mathrm{LOH})$ in a variety of tumours, including small cell lung cancer $(24,25)$, renal cell carcinoma (26), bladder cancer (27), thyroid carcinoma (28), nasopharyngeal carcinoma $(29,30)$, melanoma (31), hepatocellular carcinoma (32), breast tumour $(24)$, prostate cancer $(33,34)$ and Hodgkin's disease (35). Mutation of RASSF1A is not commonly observed.

The most frequent chromosomal loss in Chinese ESCC in Hong Kong, detected by conventional comparative genomic hybridization analysis (CGH), is 3p (46.2\%) (36). Through high-resolution array $\mathrm{CGH}(\mathrm{aCGH})$, we found that $3 \mathrm{p} 21$ is also heterozygously deleted in around half of the ESCC cell lines we studied, including both Chinese and Japanese cell lines, while homozygous deletion was not detected (Ying and Tao, unpublished data). A high frequency (72\%) of LOH at 3 p21.3 has also been described in primary ESCC from Hong Kong Chinese patients using microsatellite markers (37), suggesting that 3 p21 TSGs could also be subjected to both genetic and epigenetic inactivation in ESCC. These studies highlight the 3 p21 region as critical for ESCC pathogenesis.

As mentioned above, the incidence rate of ESCC is quite different between China and Japan, with China showing almost a three times higher rate. The possibility that different pathogenic mechanisms are operating in these two countries is appealing, but until now we have had little molecular evidence that this might be the case. RASSF 1A hypermethylation has been recently reported in Japanese ESCC by Kuroki et al $(16,38)$, but no study has been performed for Chinese ESCC. As part of our ongoing interest in the geographical distribution of disease, we investigated the epigenetic inactivation of RASSF 1A by hypermethylation in a series of Chinese ESCC cell lines and primary tumour specimens, and we report here the rather surprising results.

\section{Patients and methods}

Tissue specimens. Primary ESCC specimens were collected prospectively from 64 Chinese patients that underwent resection for ESCC since 1996 in Queen Mary Hospital, Hong Kong, and were not pretreated with chemotherapy or radiotherapy. Representative tissue samples from tumours and matching morphologically normal oesophageal epithelium tissue (nontumour) at least 6-10 $\mathrm{cm}$ away from the tumours were sampled for each patient. Sterile equipment was used for each sampling of tumour and non-tumour specimens. One half of each sample was fixed in $10 \%$ formalin for histological assessment and the other half was snap frozen in liquid nitrogen and stored at $-85^{\circ} \mathrm{C}$ for DNA and RNA extraction. For tumour samples, non-tumour portions were trimmed off from the frozen tumour blocks and the selected areas had more than $80 \%$ tumour cells as shown by histological assessment. For every 20 cryostat sections cut from the frozen blocks for DNA and RNA extraction, an additional section with hematoxylin and eosin staining was prepared for histological assessment to confirm the presence or absence of tumour cells. Six morphologically normal oesophageal epithelium samples obtained from Chinese subjects who died of traffic accidents and showed no evidence of ESCC by histology were included in this study as controls.

Cell lines. Seven Chinese ESCC cell lines (HKESC-1, HKESC-2, HKESC-3, SLMT-1, EC1, EC18, EC109) (14,39-41) and two HPV16 E6E7-immortalized normal Chinese oesophageal epithelial cell lines (NE1 and NE3) (42) were included in the study. Subsequently, we also analyzed 10 Japanese ESCC cell lines (KYSE 30, KYSE 70, KYSE 140, KYSE 150, KYSE 180, KYSE 270, KYSE 410, KYSE 450, KYSE 510, KYSE 520; obtained from the German Collection of Microorganisms and Cell Cultures).

Treatment of cell lines with demethylation agent. The demethylation treatment of selected ESCC cell lines by 5-aza2'-deoxycytidine (aza) (Sigma Chemical Co., St. Louis, MO) was carried out as described (35). The optimal concentration of aza was tested first by using 1-200 $\mu \mathrm{M}$ of aza to treat the cells over 6 days while observing cell viability. It was found that aza concentrations over $50 \mu \mathrm{M}$ were toxic to the cells. Thus, cells were diluted to $3 \times 10^{5}$ and allowed to grow overnight and aza was added to the culture medium to the desired concentration $(10 \mu \mathrm{M})$. Cells were grown for 3 days or 6 days. Fresh medium containing $10 \mu \mathrm{M}$ aza was replaced every $24 \mathrm{~h}$. After treatment, cells were pelleted and washed with phosphate-buffered saline (PBS).

DNA extraction, bisulfite treatment and methylation-specific PCR (MSP). Genomic DNA was extracted from the cell line pellets, frozen sections of paired tumour and non-tumour samples and normal oesophageal epithelium samples using proteinase $\mathrm{K}$ digestion and phenol/chloroform extraction. For each sample, 2-5 $\mu \mathrm{g}$ DNA was used for DNA bisulfite modification using the CpGenome ${ }^{\mathrm{TM}}$ DNA modification kit (Intergen Co. New York). The methylation status of the RASSF 1A promoter was analyzed using the MSP method recently developed by our group with methylated $(\mathrm{m})$ and unmethylated $(\mathrm{u})$-specific primers and 50-100 ng of bisulfite- 
a

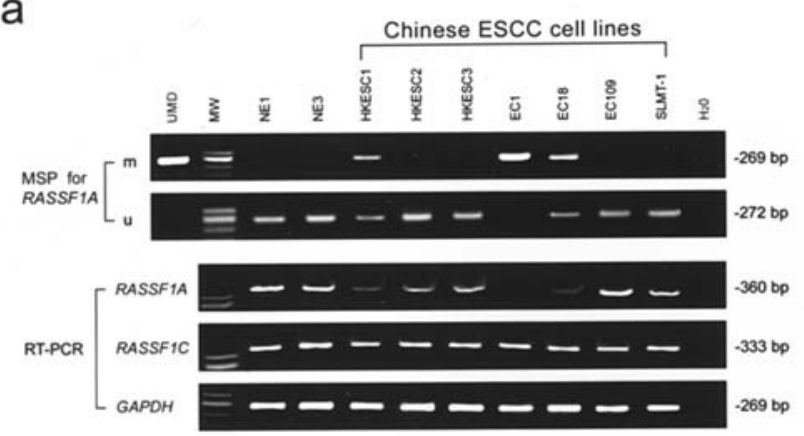

C

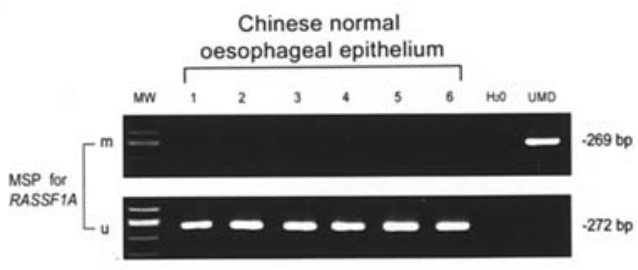

b

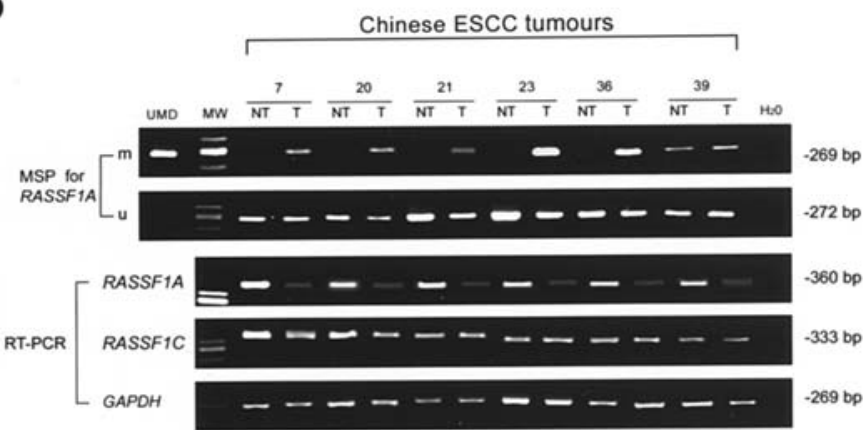

d

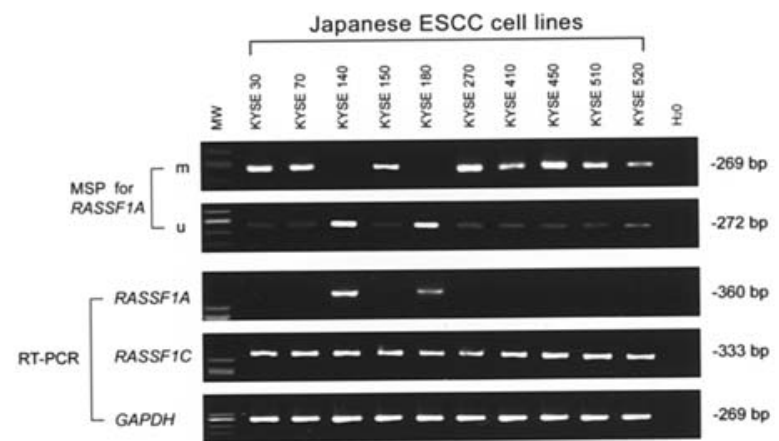

Figure 1. Analysis of the RASSF1A methylation by MSP for 30 cycles (upper panel) and RASSF1A and RASSF1C mRNA by semi-quantitative RT-PCR for 30 cycles (lower panel) in (a) 7 Chinese ESCC cell lines and 2 immortalized Chinese normal oesophageal epithelial cell lines (NE1 and NE3), (b) representative Chinese ESCC tumour (T) and non-tumour (NT) samples in cases with methylated RASSF1A in tumours (T), (c) 6 Chinese normal oesophageal epithelium samples, and (d) 10 Japanese ESCC cell lines (KYSE series). Universal methylated DNA (UMD) was used as a positive control for MSP. GAPDH amplification served as a control for cDNA quality. $\mathrm{H}_{2} \mathrm{O}$ was used as a negative control. (MW, molecular weight markers).

treated DNA (30). The MSP primers for the methylated RASSF 1A promoter were RASSF1Am1 (5'-GGT TTT TTT TAG TTT TTT TTC GTC-3') and RASSF1Am6 (5'-CTA CCG TAT AAA ATT ACA CGC G-3'), and for the unmethylated promoter were RASSF1Au1 (5'-TGG TTT TTT TTA GTT TTT TTT TGT T-3') and RASSF1Au6 (5'-ACT ACC ATA TAA AAT TAC ACA CA-3'). This MSP for 30 cycles can detect as low as $1 \%$ of methylated DNA. Universal methylated DNA (Intergen Co. New York) was used as a positive control for MSP.

Bisulfite genomic sequencing. Detailed methylation analysis of each individual site in the RASSF1A CpG island by bisulfite genome sequencing was performed as described (35). Bisulfite-treated DNA samples (50-100 ng) were amplified with strand-specific primers, BGS4 (5'-CCC RCA ACT CAA TAA ACT CAA A-3') and BGS5 (5'-GTT AAG TGT GTT GTT TTA GTA AAT-3'), which amplify a 388-bp region (-338 to +50) spanning $34 \mathrm{CpG}$ sites within the promoter and exon 1 of RASSF $1 A$ gene. PCR products were cloned into the pCR2.1-TA cloning vector (Invitrogen, Carlsbad, CA). For bisulfite genome sequencing, at least 8 bacterial colonies were analyzed for each DNA sample. Percentage methylation was determined as the percentage of methylated cytosines from 8-10 sequenced colonies.

RNA extraction and RT-PCR analysis. Total RNA was extracted from cell line pellets, frozen sections of tumours and non-tumour samples, and normal oesophageal epithelium samples using TRIzol (Life Technologies, Inc., Gaithersburg,
MD). RT-PCR for 30 cycles was performed using the GeneAmp RT-PCR kit (Perkin-Elmer-Cetus, Norwalk, CT) using $2 \mu \mathrm{g}$ RNA. The primers for RASSF $1 A$ and RASSF $1 C$ isoform were as follows: RASSF1AC (for RASSF1A, in exon 1 $\alpha$ ), $5^{\prime}-$ GAC CTC TGT GGC GAC TTC-3'; RDAA (for RASSF1C, in exon 2 $\gamma$ ), 5'-GAG GCG CCT TCT TTC GAA-3'; and RDAB (reverse primer, for both isoforms in exon 4), 5'-CAA GGA GGG TGG CTT CTT-3' (35). RT-PCR of glyceraldehyde-3phosphate dehydrogenase $(G A P D H)$ transcript was performed with the use of forward primer, GAPDH-L (5'-GGC TCT CCA GAA CAT CAT CCC TGC-3') and reverse primer GAPDH-R (5'-GGG TGT CGC TGT TGA AGT CAG AGG-3') (35). $G A P D H$ amplification served as a control for cDNA quality. $\mathrm{H}_{2} \mathrm{O}$ was used as a negative control.

Clinicopathological correlation. Statistical analysis was performed using SPSS for Windows Release 8.0.0 (22 December 1997, SPSS Inc., Chicago, IL). For analyzing correlations between categorical variables, Pearson's $\chi^{2}$ test was used. Kaplan-Meier was used to estimate survival distribution. A 2-tailed probability ( $\mathrm{p}$-value) $<0.05$ was considered as statistically significant.

\section{Results}

Analysis of RASSF1A methylation in Chinese ESCC cell lines. Analysis of RASSF1A promoter methylation in 7 ESCC cell lines and 2 immortalized normal oesophageal epithelial cell lines by MSP showed that RASSF 1A was hypermethylated in $3 / 7(43 \%)$ cell lines (HKESC-1, EC1, EC18) but not in the 
Table I. Frequency of RASSF1A hypermethylation in ESCC cell lines and tumour samples.

\begin{tabular}{lcc}
\hline Samples & $\begin{array}{c}\text { Number of } \\
\text { samples }\end{array}$ & $\begin{array}{c}\text { Detection of } \\
\text { hypermethylated } \\
\text { RASSF 1A (\%) }\end{array}$ \\
\hline Chinese ESCC & 7 & $3(43)$ \\
ESCC cell lines & 2 & $0(0)$ \\
$\begin{array}{l}\text { Immortalized normal oeso- } \\
\text { phageal epithelial cell lines }\end{array}$ & 64 & $22(34)$ \\
ESCC primary tumours & 64 & $3(5)$ \\
ESCC paired non-tumour & 64 & $0(0)$ \\
$\begin{array}{l}\text { Normal oesophageal } \\
\text { epithelium }\end{array}$ & 6 & \\
Japanese ESCC & & $8(80)$ \\
ESCC cell lines & 10 & \\
\hline
\end{tabular}

2 immortalized normal cell lines (upper panel of Fig. 1a and Table I). The unmethylated RASSFIA promoter was detected in all of the cell lines except for EC1. RNA expression of the two isoforms of RASSF1 (RASSFIA and RASSF1C) in these cell lines using isoform-specific semi-quantitative RT-PCR detected no RASSF1A mRNA in EC1 (lower panel of Fig. 1a). The 2 cell lines (HKESC-1 and EC18) which had stronger methylated and weaker unmethylated alleles, also had very low levels of RASSF1A expression. Higher levels of RASSF1A mRNA were detected in all cell lines which were unmethylated. As an internal control, RASSFIC expression was measured

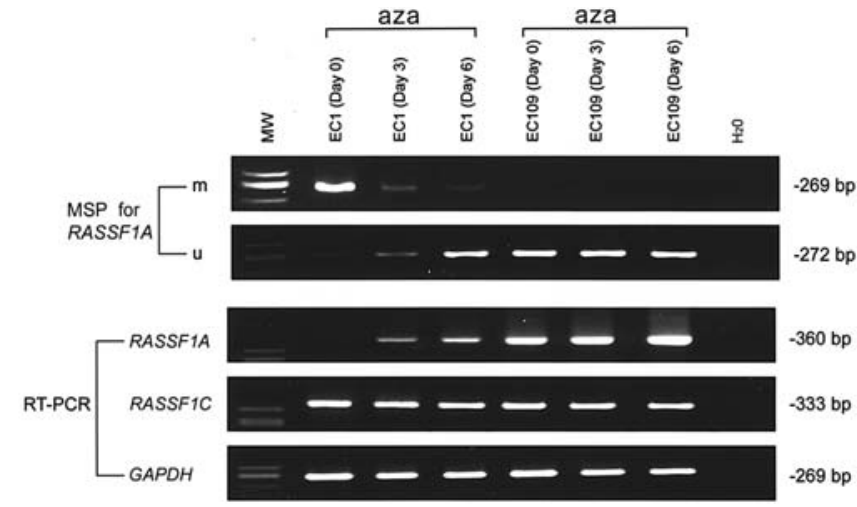

Figure 3. Induction of RASSF1A mRNA by demethylating agent aza (10 $\mu \mathrm{M})$ in the Chinese EC1 cell line with the hypermethylated RASSF1A promoter for 3 days or 6 days. The Chinese EC109 cell line with the unmethylated RASSF1A promoter was also treated as a control.

and found in all cell lines. No methylation (upper panel of Fig. 1a) and high levels of RASSF 1A mRNA were detected in the immortalized normal cell lines (lower panel of Fig. 1a).

Further bisulfite genome sequencing for RASSF1A in four ESCC cell lines (HKESC-3, EC1, EC109, SLMT-1) and one normal cell line (NE-1) showed densely methylated $\mathrm{CpG}$ sites in the RASSF1A promoter in EC1 (Fig. 2). Only scattered methylated CpG sites were detected in HKESC-3, EC109, SLMT-1, and NE-1. Bisulfite genome sequencing analysis confirmed the MSP results.

To determine whether hypermethylation of the RASSF 1A promoter directly mediates its transcriptional repression, the ESCC cell line (EC1) with complete methylation was treated with aza. A gradual restoration of RASSF1A expression was

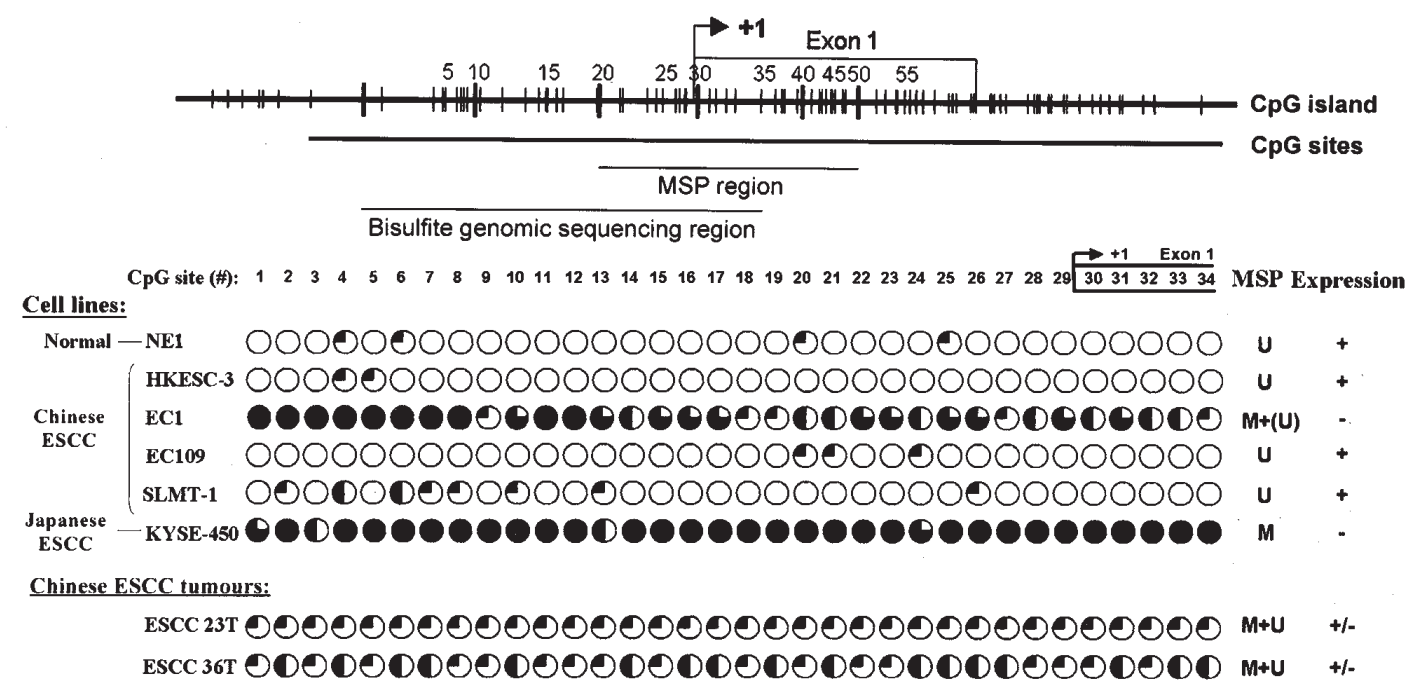

Figure 2. Detailed methylation analysis of RASSF1A by bisulfite genome sequencing of 4 representative Chinese ESCC cell lines, an immortalized Chinese normal oesophageal epithelial cell line (NE1), two hypermethylated Chinese ESCC tumour samples, and one hypermethylated Japanese ESCC cell line, KYSE 450. Top panel illustrates the CpG sites of RASSF1A and the amplified regions by MSP and bisulfite genome sequencing, covering its putative promoter and first exon, each vertical bar represents one of the $\mathrm{CpG}$ sites in the sequence. Percentage methylation was determined as percentage of methylated cytosines from 8-10 sequenced colonies. Each CpG site is shown in the row as an individual circle. M, methylated; U, unmethylated; (U), weakly unmethylated. On the right side are the MSP results and RASSF1A expression levels. 
Table II. Correlation of RASSF 1A methylation status with clinicopathological parameters in primary Chinese ESCC cases. $^{\mathrm{a}}$

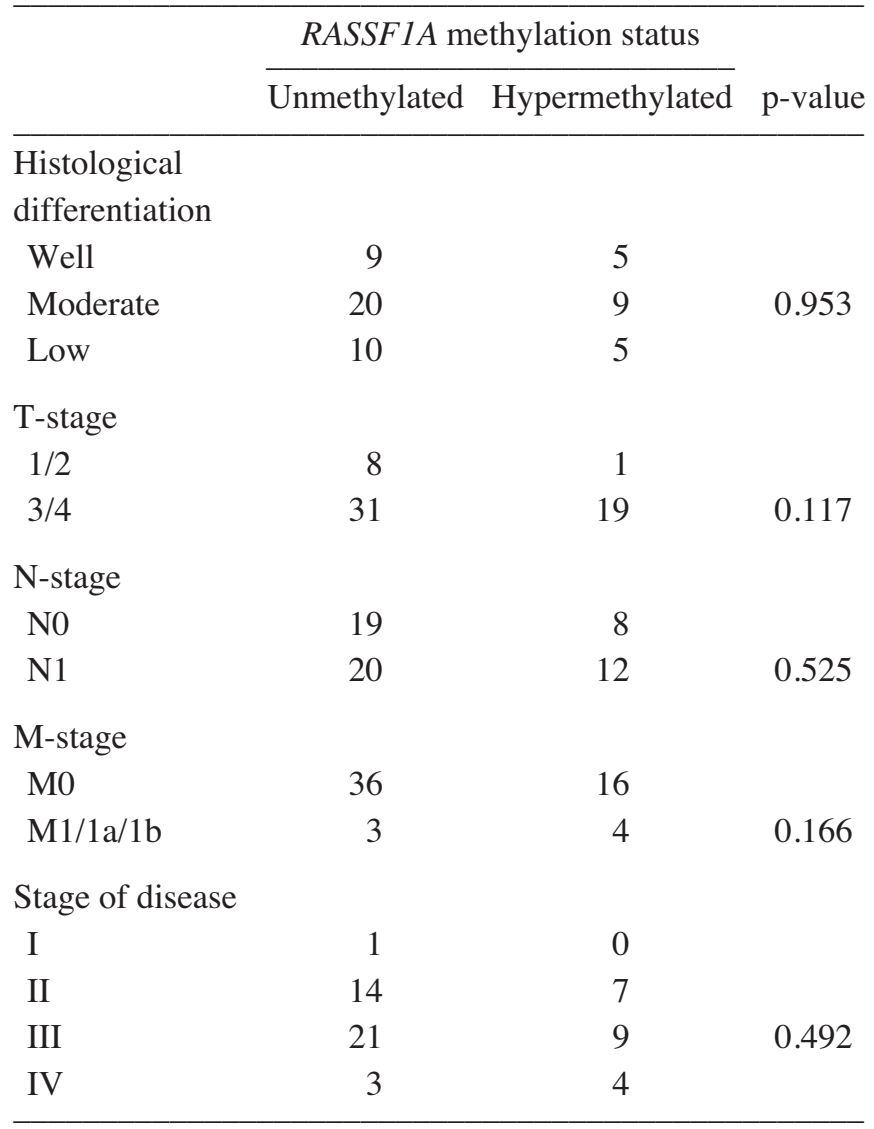

${ }^{a}$ Only 59/64 ESCC cases were statistically evaluated since complete clinical data could not be retrieved from 5 patients; $p$, Pearson's $\chi^{2}$ test.

detected after 3 and 6 days of treatment as analyzed by semiquantitative RT-PCR (lower panel of Fig. 3), concomitant with the demethylation of its promoter (upper panel of Fig. 3). Little or no change was detected in the expression of RASSF $1 C$ or the housekeeping gene, GAPDH. As a control, a RASSF1Aexpressing cell line (EC109) was also treated with aza. After 3 and 6 days of treatment, no change in the expression of RASSF 1A, RASSF1C and GAPDH was observed (lower panel of Fig. 3), and the RASSF $1 A$ methylation status was also unchanged, indicating that aza did not have an indirect effect on RASSF $1 A$ activation and demethylation (upper panel of Fig. 3). Thus, hypermethylation of its promoter directly repressed RASSF1A transcription in EC1.

Methylation of RASSF1A in clinical ESCC samples. MSP analysis of methylation in paired ESCC tumour and nontumour samples showed RASSF1A hypermethylation in 22/64 $(34 \%)$ tumour samples (upper panel of Fig. 1b and Table I). Unmethylated promoter alleles were detected in all ESCC tumour and non-tumour samples, indicating that both forms were detected and minimized the chance of false negative results. High-resolution methylation analysis by bisulfite genome sequencing of two tumours with hypermethylated RASSF $1 A$ detected by MSP confirmed that the promoter was indeed methylated in these tumours (Fig. 2), albeit only

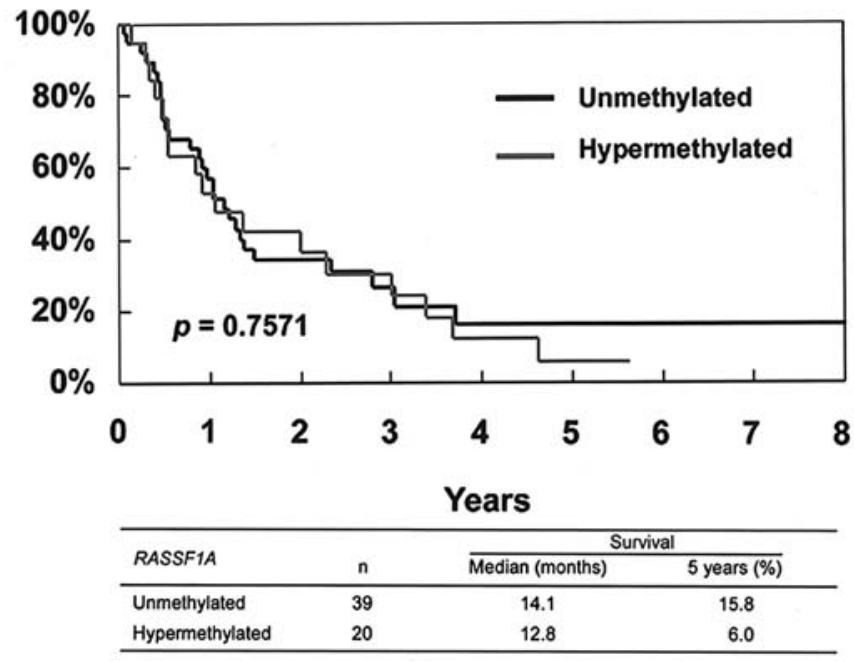

Figure 4. Correlation of survival data with RASSF1A hypermethylation in Chinese ESCC cases. Only 59/64 ESCC cases were statistically evaluated since complete clinical data could not be retrieved from 5 patients.

partially. Only three cases (5\%) showed hypermethylation in paired non-tumour tissue (e.g. no. 39). RASSF1A methylation was next analyzed in 6 normal oesophageal epithelium samples by MSP. No methylation was detected (Fig. 1c). RT-PCR analysis of paired samples showed that RASSF $1 A$ hypermethylation correlated well with mRNA down-regulation in primary tumours (lower panel of Fig. 1b). RASSF 1A was expressed in all non-tumour oesophageal tissue samples.

No correlation of RASSF1A hypermethylation with clinicopathological parameters in Chinese ESCC patients. For 59/64 cases (complete clinical data could not be retrieved from 5 patients), the relationship between RASSF $1 A$ hypermethylation and clinicopathological parameters was analyzed, including TNM staging, histological differentiation, and survival (Table II). Statistical analysis showed no significant correlation of RASSF1A hypermethylation with histological differentiation $(\mathrm{p}=0.953)$, TNM staging $(\mathrm{p}=0.117)$, or survival $(\mathrm{p}=0.7571)$ (Table II and Fig. 4).

Comparative examination of RASSF1A methylation in Japanese ESCC cell lines. Analysis of 10 Japanese ESCC cell lines using MSP showed a significantly high percentage of RASSF $1 A$ hypermethylation $(80 \%)$ in these cell lines (upper panel of Fig. 1d and Table I), compared to Chinese cell lines ( $p<0.001$, Z-test). In addition, the degree of hypermethylation also appeared to be higher than in the Chinese (Fig. 2). RASSF 1A hypermethylation correlated well with mRNA down-regulation in these cell lines (lower panel of Fig. 1d). Bisulfite genome sequencing of one of these cell lines (KYSE 450) confirmed that almost all RASSF1A alleles were hypermethylated (Fig. 2).

\section{Discussion}

Recent studies have shown that aberrant promoter hypermethylation is associated with loss of TSG functions, which promotes a cancer cell growth advantage or escape from apoptosis (43). RASSF1A is a novel candidate TSG at 3p21.3 methylated in tumours. We investigated RASSF $1 A$ methylation 
in a series of Chinese ESCC cell lines and primary tumours, and determined its relationship to clinicopathological parameters. As shown in Table I, we found that RASSF $1 A$ is silenced by promoter methylation in $43 \%$ of Chinese ESCC cell lines, $34 \%$ of primary tumours and only $5 \%$ of corresponding non-tumour oesophageal tissue, but not in any immortalized normal oesophageal epithelial cell lines and normal oesophageal epithelium samples. Our data indicates that promoter hypermethylation contributes to the inactivation of RASSF $1 A$ in a fraction of Chinese ESCC tumours.

The unmethylated RASSF $1 A$ promoter was also detected in all ESCC cell lines except for EC1. The detection of both methylated and unmethylated RASSF $1 A$ alleles in $2 / 7$ cell lines (HKESC-1, EC18) indicated a partial methylation in these cell lines, and both were found to have low levels of RASSF 1A expression. All of the resected ESCC tumours and paired non-tumour samples contained unmethylated promoter alleles, which may be explained by contributions from tumour infiltrating stromal cells. While RASSF1A methylation was not detected in any normal epithelium samples, methylation was detected in $5 \%$ of the paired non-tumour oesophageal tissue in patients with ESCC. This finding may represent the appearance of premalignant lesions or, alternatively, it could be due to the presence of some small percentage of tumour cells disseminated into non-tumour tissue, as reported in other tumours.

Recently, promoter hypermethylation of RASSF $1 A$ in Japanese ESCC has been reported in 74\% of Japanese ESCC cell lines and $52 \%$ of primary tumours $(16,38)$. A significant correlation in Japanese ESCC was found between RASSF 1A hypermethylation and tumour staging $(\mathrm{p}=0.009$, Fisher's exact test), though none was found between expression and age and histological differentiation. The proportion of Chinese ESCC cell lines and primary tumours with RASSF1A hypermethylation is roughly half of that reported in Japanese ESCC and the difference is significant ( $<<0.001$, Z-test). To investigate whether the differences observed reflected true differences between Chinese and Japanese ESCC rather than technical artefact, we subsequently analyzed 10 Japanese ESCC cell lines in a direct comparison, and detected a high percentage of RASSF 1A hypermethylation (80\%) in the Japanese ESCC cell lines. In addition, the percent of methylated sites, as determined by bisulfite genome sequencing, was also significantly different. Therefore, there are large differences between the extents of RASSF 1A hypermethylation in Chinese and Japanese ESCC. Moreover, we did not find any significant correlation between RASSF $1 A$ hypermethylation and survival rates and other clinicopathological parameters, which is in contrast to that reported for the Japanese patients. The incidence rate of ESCC is quite different in China and Japan; and our results indicate for the first time that the molecular pathogenetic mechanisms of this highly prevalent tumour could also be different in these two populations.

In conclusion, our study demonstrated that hypermethylation-associated inactivation of RASSF $1 A$ can be detected in ESCC in the Chinese but not to the same extent as is found in the disease in Japan. Our interest in how ESCC manifests differently in these two countries has been piqued, and we plan additional studies to help identify whether there is an environmental, cultural, or behavioural factor that can be modified to decrease the incidence of ESCC in China.

\section{Acknowledgements}

This work was supported by a CRCG research grant from the University of Hong Kong (to G.S.) and an A*STAR research grant to Johns Hopkins Singapore (to Q.T.).

\section{References}

1. Ferlay J, Bray F, Pisani P and Parkin DM: GLOBOCAN 2002: Cancer Incidence, Mortality and Prevalence Worldwide IARC CancerBase No. 5. Version 2.0. IARC Press, Lyon, 2004.

2. Lam KY: Molecular biology of oesophageal squamous cell carcinoma. Crit Rev Oncol Hematol 33: 71-90, 2000.

3. Tang JCO, Lam KY, Law S, Wong J and Srivastava G: Detection of genetic alterations in oesophageal squamous cell carcinomas and adjacent normal epithelia by comparative DNA fingerprinting using inter-simple sequence repeat PCR. Clin Cancer Res 7: $1539-1545,2001$.

4. Hu YC, Lam KY, Law S, Wong J and Srivastava G: Identification of differentially expressed genes in oesophageal squamous cell carcinoma (ESCC) by cDNA expression array: overexpression of Fra-1, Neogenin, Id-1, and CDC25B genes in ESCC. Clin Cancer Res 7: 2213-2221, 2001.

5. Hu YC, Lam KY, Law S, Wong J and Srivastava G: Profiling of differentially expressed cancer-related genes in oesophageal squamous cell carcinoma (ESCC) using human cancer cDNA arrays: overexpression of oncogene MET correlates with tumour differentiation in ESCC. Clin Cancer Res 7: 3519-3525, 2001.

6. Kan T, Shimada Y, Sato F, Maeda M, Kawabe A, Kaganoi J, Itami A, Yamasaki S and Imamura M: Gene expression profiling in human oesophageal cancers using cDNA microarray. Biochem Biophys Res Commun 286: 792-801, 2001.

7. Zhou J, Zhao LQ, Xiong MM, Wang XQ, Yang GR, Qiu ZL, Wu M and Liu ZH: Gene expression profiles at different stages of human oesophageal squamous cell carcinoma. World J Gastroenterol 9: 9-15, 2003.

8. Su H, Hu N, Shih J, Hu Y, Wang QH, Chuang EY, Roth MJ, Wang C, Goldstein AM, Ding T, Dawsey SM, Giffen C, Emmert-Buck MR and Taylor PR: Gene expression analysis of oesophageal squamous cell carcinoma reveals consistent molecular profiles related to a family history of upper gastrointestinal cancer. Cancer Res 63: 3872-3386, 2003.

9. Baylin SB and Herman JG: DNA hypermethylation in tumourigenesis: epigenetics joins genetics. Trends Genet 16: 168-174, 2000.

10. Maesawa C, Tamura G, Nishizuka S, Ogasawara S, Ishida K, Terashima M, Sakata K, Sato N, Saito K and Satodate R: Inactivation of the $C D K N 2$ gene by homozygous deletion and de novo methylation is associated with advanced stage oesophageal squamous cell carcinoma. Cancer Res 56: 3875-3878, 1996.

11. Tanaka H, Shimada Y, Harada H, Shinoda M, Hatooka S, Imamura $\mathrm{M}$ and Ishizaki K: Methylation of the $5^{\prime} \mathrm{CpG}$ island of the FHIT gene is closely associated with transcriptional inactivation in oesophageal squamous cell carcinomas. Cancer Res 58: 3429-3434, 1998

12. Xing EP, Nie Y, Song Y, Yang GY, Cai YC, Wang LD and Yang CS: Mechanisms of inactivation of p14ARF, p15INK4b, and pl6INK4a genes in human oesophageal squamous cell carcinoma. Clin Cancer Res 5: 2704-2713, 1999.

13. Nie Y, Yang G, Song Y, Zhao X, So C, Liao J, Wang LD and Yang CS: DNA hypermethylation is a mechanism for loss of expression of the HLA class I genes in human oesophageal squamous cell carcinomas. Carcinogenesis 22: 1615-1623, 2001.

14. Si HX, Tsao SW, Lam KY, Srivastava G, Liu Y, Wong YC, Shen ZY and Cheung AL: E-cadherin expression is commonly downregulated by $\mathrm{CpG}$ island hypermethylation in oesophageal carcinoma cells. Cancer Lett 173: 71-78, 2001.

15. Zhang L, Lu WF, Miao XP, Xing DY, Tan W and Lin DX: Inactivation of DNA repair gene $O$-6-methylguanine-DNA methyltransferase by promoter hypermethylation and its relation to p53 mutations in oesophageal squamous cell carcinoma. Carcinogenesis 24: 1039-1044, 2003. 
16. Kuroki T, Trapasso F, Yendamuri S, Matsuyama A, Alder H, Mori $\mathrm{M}$ and Croce CM: Allele loss and promoter hypermethylation of VHL, RAR-beta, RASSF1A, and FHIT tumour suppressor genes on chromosome $3 p$ in oesophageal squamous cell carcinoma. Cancer Res 63: 3724-3728, 2003.

17. Wang Y, Fang MZ, Liao J, Yang GY, Nie Y, Song Y, So C, $\mathrm{Xu}$ X, Wang LD and Yang CS: Hypermethylation-associated inactivation of retinoic acid receptor beta in human oesophageal squamous cell carcinoma. Clin Cancer Res 9: 5257-5263, 2003.

18. Yue CM, Deng DJ, Bi MX, Guo LP and Lu SH: Expression of $E C R G 4$, a novel oesophageal cancer-related gene, downregulated by $\mathrm{CpG}$ island hypermethylation in human oesophageal squamous cell carcinoma. World J Gastroenterol 9: 1174-1178, 2003.

19. Ito T, Shimada Y, Hashimoto Y, Kaganoi J, Kan T, Watanabe G, Murakami Y and Imamura M: Involvement of TSLC1 in progression of oesophageal squamous cell carcinoma. Cancer Res 63: 6320-6326, 2003.

20. Hogg RP, Honorio S, Martinez A, Agathanggelou A, Dallol A, Fullwood P, Weichselbaum R, Kuo MJ, Maher ER and Latif F: Frequent $3 \mathrm{p}$ allele loss and epigenetic inactivation of the RASSF $1 A$ tumour suppressor gene from region $3 \mathrm{p} 21.3$ in head and neck squamous cell carcinoma. Eur J Cancer 38: 1585-1592, 2002.

21. Lerman MI and Minna JD: The 630-kb lung cancer homozygous deletion region on human chromosome $3 \mathrm{p} 21.3$ : identification and evaluation of the resident candidate tumour suppressor genes. The International Lung Cancer Chromosome 3p21.3 Tumour Suppressor Gene Consortium. Cancer Res 60: 6116-6133, 2000 .

22. Dammann R, LC, Yoon JH, Chin PL, Bates S and Pfeifer GP: Epigenetic inactivation of a RAS association domain family protein from the lung tumour suppressor locus $3 \mathrm{p} 21.3$. Nat Genet 25: 315-319, 2000.

23. Hesson L, Dallol A, Minna JD, Maher ER and Latif F: NORE1A, a homologue of RASSF1A tumour suppressor gene is inactivated in human cancers. Oncogene 22: 947-954, 2003.

24. Burbee DG, Forgacs E, Zochbauer-Muller S, Shivakumar L, Fong K, Gao B, Randle D, Kondo M, Virmani A, Bader S, Sekido Y, Latif F, Milchgrub S, Toyooka S, Gazdar AF, Lerman MI, Zabarovsky E, White M and Minna JD: Epigenetic inactivation of RASSFIA in lung and breast cancers and malignant phenotype suppression. J Natl Cancer Inst 93: 691-699, 2001.

25. Dammann R, Takahashi T and Pfeifer GP: The CpG island of the novel tumour suppressor gene RASSF $1 A$ is intensely methylated in primary small cell lung carcinomas. Oncogene 20: 3563-3567, 2001.

26. Dreijerink K, Braga E, Kuzmin I, Geil L, Duh FM, Angeloni D, Zbar B, Lerman MI, Stanbridge EJ, Minna JD, Protopopov A, Li J, Kashuba V, Klein G and Zabarovsky ER: The candidate tumour suppressor gene, RASSF1A, from human chromosome 3 p21.3 is involved in kidney tumourigenesis. Proc Natl Acad Sci USA 98: 7504-7509, 2001.

27. Lee MG, Kim HY, Byun DS, Lee SJ, Lee CH, Kim JI, Chang SG and Chi SG: Frequent epigenetic inactivation of RASSF $1 A$ in human bladder carcinoma. Cancer Res 61: 6688-6692, 2001.

28. Schagdarsurengin U, Gimm O, Hoang-Vu C, Dralle H, Pfeifer GP and Dammann R: Frequent epigenetic silencing of the $\mathrm{CpG}$ island promoter of RASSF1A in thyroid carcinoma. Cancer Res 62: 3698-3701, 2002.

29. Kwong J, Lo KW, To KF, Teo PM, Johnson PJ and Huang DP: Promoter hypermethylation of multiple genes in nasopharyngeal carcinoma. Clin Cancer Res 8: 131-137, 2002.
30. Qiu GH, Tan LKS, Loh KS, Lim CY, Srivastava G, Tsai ST, Tsao SW and Tao Q: The candidate tumour suppressor gene $B L U$, located at the commonly deleted region $3 \mathrm{p} 21$, is an E2Fregulated, stress-responsive gene, and inactivated by epigenetic and genetic mechanisms in nasopharyngeal carcinoma. Oncogene 23: 4793-4806, 2004.

31. Spugnardi M, Tommasi S, Dammann R, Pfeifer GP and Hoon DS: Epigenetic inactivation of RAS association domain family protein 1 (RASSF1A) in malignant cutaneous melanoma. Cancer Res 63: 1639-1643, 2003.

32. Schagdarsurengin U, Wilkens L, Steinemann D, Flemming P, Kreipe HH, Pfeifer GP, Schlegelberger B and Dammann R: Frequent epigenetic inactivation of the RASSF 1A gene in hepatocellular carcinoma. Oncogene 22: 1866-1871, 2003.

33. Kuzmin I, Gillespie JW, Protopopov A, Geil L, Dreijerink K, Yang Y, Vocke CD, Duh FM, Zabarovsky E, Minna JD, Rhim JS, Emmert-Buck MR, Linehan WM and Lerman MI: The RASSF1A tumour suppressor gene is inactivated in prostate tumours and suppresses growth of prostate carcinoma cells. Cancer Res 62: 3498-3502, 2002.

34. Liu L, Yoon JH, Dammann R and Pfeifer GP: Frequent hypermethylation of the RASSF $1 A$ gene in prostate cancer. Oncogene 21: 6835-6840, 2002.

35. Murray PG, Qiu GH, Fu L, Waites ER, Srivastava G, Heys D, Agathanggelou A, Latif F, Grundy RG, Mann JR, Starczynski J, Crocker J, Parkes SE, Ambinder RF, Young LS and Tao Q: Frequent epigenetic inactivation of the RASSF 1A tumour suppressor gene in Hodgkin's lymphoma. Oncogene 23: 1327-1332, 2004.

36. Kwong D, Lam KY, Guan X, Law S, Wong J and Sham J: Chromosomal aberrations in oesophageal squamous cell carcinoma among Chinese: gain of $12 \mathrm{p}$ predicts poor prognosis after surgery. Hum Pathol 35: 309-316, 2004.

37. Ko JM, Wong CP, Tang CM, Lau KW and Lung ML: Frequent loss of heterozygosity on multiple chromosomes in Chinese oesophageal squamous cell carcinomas. Cancer Lett 170: 131-138, 2001.

38. Kuroki T, Trapasso F, Yendamuri S, Matsuyama A, Alder H, Mori $\mathrm{M}$ and Croce CM: Promoter hypermethylation of RASSF1A in oesophageal squamous cell carcinoma. Clin Cancer Res 9: 1441-1445, 2003.

39. Hu Y, Lam KY, Wan TS, Fang W, Ma ES, Chan LC and Srivastava G: Establishment and characterization of HKESC-1, a new cancer cell line from human oesophageal squamous cell carcinoma. Cancer Genet Cytogenet 118: 112-120, 2000.

40. Tang JC, Wan TS, Wong N, Pang E, Lam KY, Law SY, Chow LM, Ma ES, Chan LC, Wong J and Srivastava G: Establishment and characterization of a new xenograft-derived human oesophageal squamous cell carcinoma cell line SLMT-1 of Chinese origin. Cancer Genet Cytogenet 124: 36-41, 2001.

41. Hu YC, Lam KY, Law SY, Wan TS, Ma ES, Kwong YL, Chan LC, Wong J and Srivastava G: Establishment, characterization, karyotyping, and comparative genomic hybridization analysis of HKESC-2 and HKESC-3: two newly established human oesophageal squamous cell carcinoma cell lines. Cancer Genet Cytogenet 135: 120-127, 2002.

42. Deng W, Tsao SW, Guan XY, Lucas JN, Si HX, Leung CS, Mak P, Wang LD and Cheung AL: Distinct profiles of critically short telomeres are a key determinant of different chromosome aberrations in immortalized human cells: whole-genome evidence from multiple cell lines. Oncogene 23: 9090-9101, 2004.

43. Herman JG and Baylin SB: Gene silencing in cancer in association with promoter hypermethylation. N Engl J Med 349: 2042-2054, 2003. 